\title{
How Technology Helped Create National Standards for Early and Special Needs Education
}

\author{
Jolanta Galecka \\ Young Digital Planet
}

\begin{abstract}
Special Needs require special attention. Individualized attention. They need to be understood and catered to. They are a part of early education. Early Education lays the grounds for future development. It needs to be regarded with utmost attention. Young Digital Planet started to create applications that were designed to help children restore their curiosity and keep learning engaging, motivating and challenging, long time ago. One of those products was eduSensus. Originally based on intuition, designed for speech therapists and combined with the educational needs communicated by the market, eduSensus has grown from a few original applications to a versatile and comprehensive family of software supported by specially designed hardware and traditional materials. The adaptation and adjustment to the needs of the market was a result of the response from the users: the educational needs were far greater than we had anticipated. The product affected the therapy process, established new trends and eventually created new standards in speech therapy. As it turned out, children born after 1993 did not know a world without a computer, so learning without it seemed out of their world.
\end{abstract}

\section{Introduction}

If you talk to teachers nowadays and ask them how they perceive their students, they will - most likely - tell you that the level of children's development has decreased. That the number of students and the diversification of the class make the teaching process very hard, almost impossible. What is more, the number of children with disorders seems to be growing and there are more and more children classified as having Special Needs. The discussion over the Ofsted (Office for Standards in Education) major review of special educational needs and disability arrangements revealed that there is a serious problem not only with the classification but also with the definition of Special Needs [1]. But there is one common voice coming out of all that commotion: Special Needs and Early Education NEED special attention.

So what seems to be wrong?
We are educating our children too late or not adequately and sufficiently enough. Children have an amazing potential and the earlier we start the bigger the potential. But I am not talking about teaching children to read and write when they are 2 years old. Quite the opposite - I am talking about an overall enhancement of all the developmental skills, adjusted to the pace of the individual path of a particular child. The way eduSensus has been prepared.

The software - originally developed for the specialist market of therapists - has been in use in Poland for almost 10 years now and has proven to be an irreplaceable tool for early education and special needs prevention.

Because Special Needs can be - in many cases prevented if we start the education process early enough:

Technology enables attention to the needs of the children, individualization and special catering. Yet, even though the technology had been present for some time, there was no change in the approach towards early and special needs education. Technology was treated as unnecessary evil by some or by threatening obstacle or another difficult task one is forced to accomplish. But many saw what technology was really good for. They saw its potential and the chance it gives to transform the education. To implement all those solutions that many experts and scientists have been talking about for decades. Young Digital Planet was among them.

\section{Literature Review}

Children learn by watching, listening, babbling, imitating, analyzing, playing, repeating, trying, interacting with the world and manipulating it [2]. They should be allowed to follow that path at their own pace but they should also be guided properly and provided with the necessary tools. Children like to pursue the subject of their interest in depth and they like to have some control over that pursue. They experiment, make mistakes and learn from them. The child is ready to accumulate, associate and transform the inputs they receive from the world but they are also ready and prepared to receive help from adults. And the adults are naturally equipped to give that help [3]. It's just that recently they do not have enough time and resources for the individual 
attention and maybe this is the reason for the recent decline.

If a child's development is not stimulated, they may never have the opportunity to fully develop their potential. The adults need to guide the play, to lead the path. A child left to their own devices will develop certain skills but they will not develop all areas and skills required later on in the next stage for example [4]. The child will feel anxious and will lose the necessary self-esteem. The progress will be impeded and the damage done much harder to repair. Certain skills like hearing sensitivity, music, speech, motor coordination and audio coordination can be shaped in one way only - through hundreds of repetitions. Making all the areas of development attractive for a child and remembering about all of them is the base for a thorough and comprehensive development [5]. Children are naturally very curious and they like to learn. That is how human beings have been designed in order to survive. We adapt to changing environment. We get smarter and we enjoy it. Play needs to be a part of that learning process as it is a natural part of our existence. Children deprived of play completely always suffer developmental challenges. But even learning through play needs to be coordinated, stimulated and guided [6].

Why do so many kids today have problems at school? Because some areas of their development have not been stimulated, have been neglected. The child was left to their own devices or there was not enough motor stimulation or their parents simply did not have the time to read to a child on a regular basis. Since imitation is such an important vessel for gaining knowledge, the child needs to be exposed to enough stimuli and modeling to be able to understand it, embrace it and internalize it.

\section{Research Rationale}

Since Early and Special Needs Education are of such vital importance, we cannot simply provide random stimulations embedded in play. We need to ensure to do it right. And for that one has to have a certain amount of knowledge about child development, the versatile media formats and the methods of teaching. Almost 20 years of experience has given Young Digital Planet the necessary knowledge. The secret lies in the individualisation of the educational process. One teacher cannot possibly teach each child separately if the class is bigger than 4-5 students. Unless...

Unless the teacher is provided with the necessary tools. Tools that will allow a better recreation of reality. Multimedia and technology provide us with a chance to bring the real world to the student's desk. Alive and buzzing, just as it would be if it was actually happening. And what is most important that technology will allow students to interact with the reality, not just watch it. Traditional books are still and the world around us is not. Movies get closer to reality but they can only be watched, which - as it turns out - puts our brain cells in "sleep mode". And there is also another issue - trust. Children need to believe in what they are taught. Just like they believe in the power of Google... Since computers and mobile devices are what they are surrounded by, they need to be the carriers of education as well. Guided by thoughtful but technologically savvy teachers.

Young Digital Planet has realized early on that Early Prevention is of utmost importance. The education of children between 0 and 6 years old lays the foundations for their future development. And by education I mean fun but wise and versatile play. Learning that's engaging and does not kill the natural curiosity in children. Learning that is meaningful and takes into account the developmental stages of the child. During those early stages children often need to deal with certain difficulties. The better adjusted the learning to the individual needs of a particular child, the less of those difficulties the child will encounter. This is very important if we want the children to develop into curious and creative adults. Their desire to learn needs to be nurtured and cared for.

It is even more important now - since the civilization changed the way we interact with the world. Visual images are replacing all the other channels of communication. Our brain has not been designed for that. A child needs to interact with the world, not watch it. The need for reading is declining, the need for any kind of effort is diminishing, everything is readily available and children are getting used to instant gratification. Even the need to go out and meet other people seems to be disappearing in some families. And our brain is very social. It needs this type of stimulation. We need to remember how we developed: children interacted with a lot of adults who took care of them. They lived in big communities, where every member was connected to the others. And now there seems to be a shortage of time and human resources for such interaction. The parents' engagement has decreased and that has impacted the emotional development, which in turn impacts the memory and the ability to learn in general.

We can change that: every minute spent with a child on wise play is the best time investment possible.

Designing a product for early and special needs education we wanted to cover all the areas of child development to make sure nothing has been left out. We based it on ontogenetic language development and addressed various learning difficulties. The education process is fun and provides endless opportunities for repetition. We also needed to connect the two worlds: the traditional and the digital. And so hundreds of printable materials, 
videos with physical exercises to follow, musical activities to perform, recitation and even sign language to stimulate all areas of the brain were included. It is a completely different approach to education, one that takes the individuality of each child into account.

\section{Special Needs}

More and more students are having trouble acquiring even the basic skills. Teachers are searching for new strategies, approaches and techniques that would meet the needs of the learners. Especially, that some of the tried (and still used) strategies are not as successful as they used to be.

Learning difficulties occur when a child is unable to perform a developmental task placed at a particular age level. Very often the psychological needs of the children are not satisfied. Often it is the way the task is presented or the environment it is presented in. The brain experiences difficulties in bringing together information from its different regions. And we need to remember that gifted children may also have learning disabilities.

Identifying and helping learners with their difficulties has never been easy. The biggest challenge is to identify the right learners as early as practically possible. The research indicates that controlling task difficulty (with assistance), working in small groups and using structured questioning prove to be highly effective for learning difficulties. It is also important for the teachers to model problem solving. Direct instruction should also be combined with strategy instruction, where learners can understand how to learn.

Inclusive education seems to be a developing trend as more and more students with learning disorders are placed in regular classes. This of course increases the responsibility of the teachers. Unfortunately very often the inclusive education has little to do with true inclusion as the children are assigned a separate teacher or caretaker and kept aside of the rest of the group. They are also provided with different materials.

There are many misconceptions regarding learning disabilities. Not long ago we knew very little about the causes of learning disorders. Science made a huge progress. The developing technology made it possible to look inside the brain and learn its structure and how it works. This gives us a chance to design techniques that will be successful not only at addressing the brain disorders but also at preventing at least some of them. Technology allows teacher to engage with Special Needs students in new ways.

There is also another issue we should be aware of: there are many students who are labeled as disabled when in fact they are just struggling to follow the strategies prevalent in traditional schooling. Majority of schools have not changed their approach to education, regardless of the scientific discoveries and rapidly developing technology. As it turned out the speech therapy for example had not changed much either. At least not in Poland. So there was a big gap between what science knew and what the practice was actually doing.

Reading for example requires doing many things at once with precise timing. Children who have dyslexia have trouble with phonemic awareness, which means the ability to hear, identify, and use each individual sound by manipulating the sounds. Children begin to read as they learn the speech sounds that make the words and connect them to alphabet letters. Children blend the sounds into words (eventually recognizing the words instantly) and when the process becomes automatic they may concentrate on the content and meaning of what they are reading. When the information processing is disturbed then the process of reading does not become automatic and the comprehension suffers. Children with dyslexia do not rapidly recognize the words. The earlier it is diagnosed and treated the lesser the chance that the reading problem will impede other areas that rely on the comprehension of the text. The child self-esteem will not suffer as much.

A very important part of every diagnosis is asserting, what causes the difficulties. They may be diverse and the specialist should be able to discover them and find out whether they are of an environmental, psychological or biological nature. The diagnosis should describe the situation that causes the difficulties in different environments of the child's life. It should also be able to point out the dysfunctions that lay at the core of the problem. But an incredibly important part of the diagnostician's task is to uncover the potential of the child, show their strong features and aptitudes.

Now, with the recent developments in the diagnosis of dyslexia it is common knowledge that the problems with speech, reading and writing are related to the coordination of the whole body and its motorics as well as with visual and musical skills. The statement that we only use $10 \%$ of our brain is a myth. Whatever we do many parts of the brain are at work and engaged and it is important for all of them to work properly and to be developed comprehensively. Therefore, when teaching languages for example, we must not forget graphomotorics, thinking skills, simple clauses, complex instructions, dialogues, songs and poems, antonyms, colours and sequences. The suprasegmental qualities of speech should be shaped as well as the motorics and coordination. They are all equally important. Learning sounds should be associated with visual aspects and learning new words should be associated with audio aspects. And if all this can be placed in its natural environment to reduce the need for guessing what it actually is, so 
much the better. So this is exactly what we decided to do.

\section{Language Development}

Language development is one of the most important aspects of the child's development. It allows for communication, environment comprehension and interaction.

First we need to be able to utter sounds at all, then practice our auditory sensitivity and realize that we have control over our own voice. We need to practice articulation, focus on the suprasegmental language features and proper voice projection. We need activities for the improvement of articulators, shaping the proper prosodic speech qualities and supporting the appropriate vocal emission, controlling the rhythm, pace, volume, level of the voice, modulation, intonation, and lengthening of the expiratory phase. We need the environment that will develop the skills of: auditory perception, visualmotor coordination and auditory-motor coordination.

Then the child needs to further exercise the vocal organs and develop linguistically, starting from imitating sounds and differentiating between them. Onomatopoeic expressions in the first stage are the best. This allows to train all vocal functions:

breathing, phonation (voice), prosody, phonemic hearing, efficiency and coordination of vocal organs and articulation. Mastering other concepts such as spatial relations, antonyms, names of the colours, musical instruments, vehicles, animals, natural phenomena enhances the process of absorption..

And we cannot forget about supporting the child's development in such fields as: development of the auditory perception, sound differentiation, exercising memory and auditory sensitivity, logical thinking, articulators, phonological awareness, matching and sorting exercises, improving left-right orientation, widening passive and active lexical capacity. Additional features such as voice recording make the process even more efficient as they allow comparison with the original sounds.

Then we also need to put emphasis on the process of language acquisition as well as its proper development. Lexical, grammatical, expressive and pragmatical aspects of language cannot be omitted. Free communication with the child's immediate environment is the base of every language acquisition. To enable the child to understand others and to be understood we need to let them shape the skill of understanding speech and allow them to speak their mind. Supporting the development of the following language aspects: semantic-lexical, morphological-syntactic and pragmatic will be of highest importance.

But we cannot forget about shaping other skills like: social skills, Audio-visuo-motor coordination, Auditory perception, Mental skills, Spatial perception, Language skills and phonological awareness.

While shaping the free communication we also need to work on expanding a child's knowledge of vocabulary in the specific lexical categories and mastering communication skills and competence. Improving a child's passive and active vocabulary through adding new items to a child's lexical store. Creative work - like building a comic for example will allow to check whether the child uses vocabulary in a logical and comprehensive manner.

Language and music are closely correlated. Influence of music and music therapy should be used to improve or correct language communication as well as prevent delays in this area, enhance the speech abilities and especially to practice suprasegmental aspects of the speech. This can additionally act as support in the comprehensive development of the child through motor, auditory and verbal-musical stimulation. Exercises in:

- big motor activity

- basic locomotive movements

- motor coordination combined with visual and auditory coordination

- auditory perception

- pace differentiation

- rhythm, accent, metre, timbre, articulation and melody of sounds and

- graphomotorics

should be supported. Sensitivity to sound stimuli should be increased, audio-motor coordination developed and speech fluency practiced.

Just like in a healthy environment children should acquaint themselves with theoretical and practical aspects of music, create their own melodies, play with the musical scale and rhythmical values.

But above all personalization should be enabled. Because the experiences of every individual are different and they vary considerably, so a one-cut education is never going to be really successful. When all those aspects are combined we can talk about a natural approach to learning in general. Such an approach indeed gives a chance for success.

Thanks to technology such an approach was implemented in Young Digital Planet and all of the above aspects of language development have been included in the eduSensus products.

\section{6. eduSensus}

eduSensus series is a family of products addressing all developmental needs. One of the eduSensus advantages is that it has been created together with therapists, teachers, parents and children. This resulted in the building of a learning environment that hardly seems educational. Set in natural environment, with activities that seem only natural, working with eduSensus seems like spending 
a regular day outside school rather than actually learning.

The input is given in a meaningful situation and surrounding, combined with audio and video reinforcement. It is a perfect way to acquire comprehension. There are many commands that require physical movement. There is a whole chapter of physical exercises included within the activities.

The number and variety of the activities allow for a full behavioral approach, where natural life situations are being used. The lesson scenarios that may be created on the base of eduSensus material enable a full spectrum of functions to be applied, where children can learn how to express their needs, desires, suggestions, follow orders, express feelings and construct dialogues.

But what's most important is the ability to individualize the process of learning, to build it in such a way that allows for the free choice of activities and for their countless repetitions. A true autonomy in learning is therefore possible. Children can choose what they are interested in and follow that interest. The atmosphere of the activities is relaxed and errors are not punished. The activities allow for their verification, to show whether the path the child has chosen is a correct one. Amazing audio and visual feedback in eduSensus provide just that.

After almost 10 years of development eduSensus consists now of huge module for Speech Therapy, another module for Pedagogical Diagnosis and Therapy (including Dyslexia, Intelligent Pen with Tablet and Math), Developmental Scaffolding Module (including Development Assistant, School Readiness, Gifted \& Talented), Equal Opportunities Module (including Mouthmouse and Tomatis Method).

\section{Creating National Standards}

The whole idea started as a therapy product for speech and language pathology only to later develop into a complex and comprehensive product used for the therapy of various intellectual and motoric impairments, early prevention as well as the enhancement of early development and even for language learning. The most difficult task was to design a unique methodology and an innovative learning environment together with attractive content and design to create an engaging world for children to enjoy and benefit from what was familiar enough to feel natural and non-invasive.

The therapists were used to old-fashioned, traditional methods, based on pen and paper. When they saw the possibilities that technology had brought to their profession they immediately realised its potential. They saw immediate results in their patients, especially in their attitude towards therapy sessions and learning in general. And the engagement is crucial here - and therapists are constantly searching for resources.

Therapy is a chore, a fight with the child's problems, it can be depressing. The kids are nervous, they are afraid of failure especially when they are well developed (with no intellectual delays) and only have minor speech problems. Instead of having to talk to the mirror they suddenly saw a funny clown or cannon or something else colourful and interesting. And therapists have started to communicate their needs. The ontogenetic development of the natural language was combined with the methods for learning a second language. All the activities were based on skills and not information. It is important to shape a child's thinking skills and not just feed them with new facts to test them later on. All aspects of uninhibited natural child development need to be included.

It seems that the secret lay in the approach: we adjusted to the world of the children, thinking about the way they develop naturally (without the interference of teachers, so to speak), what triggers their interest and what doesn't. Then we listened to their feedback and we tried to adjust. But most importantly we - by wanting to be innovative and modern - offered the children the world they knew best: interactive and digital. Children do not know the world without computers. For them it is not an innovation, it's simply the reality they know how to interact with.

We have encountered many problems: technological advancement was a barrier, voice calibrators were not adaptable to every computer, sound recording was not compatible. The perception of graphics and animations by the physically impaired children was different than the approach of our graphic designers, so we needed to make a lot of changes. Computer skills of the users were also an issue. Basic computer skills needed to be taught within the application. A scanning option had to be implemented. The colours of letters and their background had to be added to help dyslexic children to read them. The seasons were used as a therapy tool and an attachment to reality was one of the main features of the product. Phonetic pureness was developed and became a standard.

This approach allowed for a versatile use of the product: eduSensus was used by the developmentally impaired children and as a developmental enhancement by children without any impairment. Due to its design it could be personalized to any needs. Therapy and learning finally became engaging and fun. The natural approach, the ontogenetic language development and the relation with the environment made its use versatile and adaptable.

The product received numerous awards. 100\% Pedagogical and Psychological Centres in Poland use eduSensus. eduSensus programs were included in the list of didactic aids recommended by the Polish 
Ministry of Education (since 2008 the MoE can recommend workbooks only). The series has also been approved as a medical device: it complies with the requirements of Medical Device Directive 93/42/EEC and its equivalent - the Polish norm PNEN ISO 13485:2004. eduSensus medical device has been awarded the $\mathrm{CE}$ label thanks to the implementation of the ISO 13485 Quality Management System.

The number of therapists who work in schools is growing. The number of therapists in preschools is not known in Poland. Special needs therapists offer mainly private services for preschool children and parents pay for the therapy. Sometimes preschool teachers provide special needs support for children with disorders. The importance of early education and early prevention should definitely be highlighted in this market sector.

As a result of the market demand additional modules have been added to speech and language therapy and new ideas for therapy enhancement appeared: 'Dyslexia', 'Gifted \& Talented' and 'School Readiness' - enhancement and assessment.

\section{Conclusions}

For example, "1. Introduction", should be Times 12-point boldface, initially capitalized, flush left, with one blank line before, and one blank line after. Use a period (“.”) after the heading number, not a colon.

It all started with hunches and feelings and impressions and was based on intuition. However, that approach was only possible thanks to the fact that the people whose hunches and intuition we relied on, were true experts dealing with children on a daily basis and - most importantly - taking the children's interest seriously and willing to change the approach when a different need was communicated.

But technology is still often feared by many people. They are afraid to trust something they do not know or understand. We noticed that the only effective way to inform the people in the institutional society like preschools or therapists was to create an Academy. The Young Digital Planet Academy is a group of people called "trainers" who know the product inside out and have a good methodological background. It could be somebody who has worked with children and is familiar with the learning methods. They should be a scientific soul as well. Teachers and principals don't like offensive promotion so we prefer giving lectures on ICT, child development and new methods of learning with the eduSensus examples serving as the background. Some teachers are afraid of using computers and the Internet. They will be more eager to use the products when they familiarize themselves with technology first and with the product itself. YDP Academy shows how to use the product and provides useful information on ICT and new teaching methods.

The workshops by the eduSensus Team cover the following topics:

- The use of ICT tools in supporting the development of SEN children.

- The group of dyslexia risk: early diagnosis, prevention and stimulation of development with the use of ICT tools.

- Effective and engaging stimulation of retarded children's development.

- Innovative speech therapy - how to enrich your techniques with new technologies.

- A child ready for school. Diagnosis and early support of child development.

- A happy kindergarten without student books. How ICT can help children.

- Developing young learners' vocabulary and communicative skills with digital programs.

- Developing social skills in autistic children using educational software.

- Interesting ways of working with children with graphomotor skills deficiency.

- The modern common room - a friendly place to learn and have fun in.

- How to introduce children into the IT world. The computer in the early years of primary education.

After every workshop each participant receives a confirmation of training completion. If the participant completes at least 5 of the 11 training modules, they are awarded a Certificate of YDP Academy for Innovative Teachers.

Technology gives us an amazing opportunity to recreate natural environment - the one that humans have always learnt in and benefited from the most. When the generation of digital natives gets to the decision making positions there will be no questioning or hesitation about the implementation of all the technology into education. But the digital immigrants now have this unique opportunity to combine the knowledge gained through traditional learning, including the values, the pace, the relevance and the quality, and transfer some of it (hopefully the best parts) into the digital world. That is why it is so important not to concentrate on technology environments and tools only but to lay the grounds for new learning. The grounds that will be accepted by the new generation. We should use the new technology to achieve what we now realize we have been lacking: personalization, attention to special needs, a natural approach to learning and the reduction of the administrative burden. New technology environments will give it all a proper shape and will enable us to reach out towards the digital natives. Because they will not accept any other form. 


\section{References}

[1] Ofsted "The special educational needs and disability review", http://www.ofsted.gov.uk/resources/specialeducational-needs-and-disability-review

[2] Alison Gopnik, Andrew Meltzoff, Patricia Kuhl "The Scientist in the crib” Harper Parennial 2001

[3] Jane M. Healy "Your child growing mind", Broadway Books 2004

[4] John Holt "How children learn”, Penguin Books 1991

[5] Edward de Bono "Teach your child how to think", Helion 2010

[6] Stuart Brown "Play. How it shapes the brain, opens the imagination and invigorates the soul" Avery 2010 